\title{
ÜBER DAS VERHALTEN DER LÖSUNGEN DER MAXWELLSCHEN RANDWERTAUFGABE IN EINIGEN NICHTGLATTEN GEBIETEN
}

\author{
JUKKA SARANEN
}

Sei $\Omega \subset \boldsymbol{R}^{3}$ ein Gebiet. Die zeitunabhängige Maxwellsche Randwertaufgabe für isotrope homogene Medien lautet

$$
\left\{\begin{array}{l}
\nabla \times E-i \omega \mu H=J \\
\nabla \times H+i \omega \varepsilon E=K \\
n \times\left. E\right|_{\Gamma}=0
\end{array}\right.
$$

mit $\Gamma=\partial \Omega$. Dabei sind $\varepsilon$ und $\mu$ positive Materialkonstanten, und $\omega$ ist eine komplexe Zahl. Führt man in (0.1) die Substitutionen $H=\sqrt{\bar{\varepsilon}} H^{\prime}, E=\sqrt{\mu} E^{\prime}$, $\omega \sqrt{\varepsilon \mu}=\omega^{\prime}$ ein, so ergibt sich

$$
\left\{\begin{array}{l}
\nabla \times E^{\prime}-i \omega^{\prime} H^{\prime}=\mu^{-1 / 2} J \\
\nabla \times H^{\prime}+i \omega^{\prime} E^{\prime}=\varepsilon^{-1 / 2} K \\
n \times\left. E^{\prime}\right|_{\Gamma}=0
\end{array}\right.
$$

Deswegen setzen wir im folgenden o. B. d. A. $\varepsilon=\mu=1$ voraus. Bei der Behandlung von (0.1) mit Hilbertraum-Methoden ist es üblich die Räume $R(\Omega)=\left\{E \in L^{2}(\Omega)^{3} \mid\right.$ $\left.\nabla \times E \in L^{2}(\Omega)^{3}\right\}, \quad D(\Omega)=\left\{E \in L^{2}(\Omega)^{3} \mid \operatorname{div} E \in L^{2}(\Omega)\right\}, \quad R^{0}(\Omega)=\{E \in R(\Omega) \mid(\nabla \times E \mid F)=$ $(E \mid \nabla \times F) \forall F \in R(\Omega)\} \quad$ zu benutzen. Die schwache Form von $(0.1)$ wird $(\varepsilon=\mu=1)$

$$
\begin{cases}\nabla \times E-i \omega H=J, & E \in R^{0}(\Omega), \quad H \in R(\Omega), \\ \nabla \times H+i \omega E=K, & J, K \in L^{2}(\Omega)^{3} .\end{cases}
$$

Die Existenztheorie der Aufgabe (0.2) ist weit entwickelt worden [5], [6], [16], [17], [18]. Für eine umfangreiche Klasse von Gebieten hat man die Fredholmsche Alternative bewiesen. Dasselbe gilt auch für anisotrope inhomogene Medien [16], [17]. Von den qualitativen Eigenschaften der Lösungen ist im wesentlichen folgendes bekannt. Falls die rechte Seite die zusätzliche Regularität $J \in R(\Omega), K \in D(\Omega)$, $\operatorname{div} K \in H_{0}^{1}(\Omega)$ erfüllt, kann man das Feld $H$ aus $(0.2)$ eliminieren $(\omega \neq 0)$, und man erhält die elliptische Gleichung

$$
-\Delta E-\omega^{2} E=F, \quad E \in R^{0}(\Omega) \cap D(\Omega), \quad \operatorname{div} E \in H_{0}^{1}(\Omega)
$$


mit $F=\nabla \times J+i \omega K+i \omega^{-1} \nabla$ div $K$. Nach einem bekannten Verfahren [1] schließt man dadurch in glatten Gebieten auf die übliche Regularität $E \in H^{2}(\Omega)^{3}$ mit

$$
\|E\|_{2} \leqq C(\|E\|+\|F\|)
$$

[8], [9]. In nichtglatten Fällen ist die Situation anders. Die Lösungen von $(0.3)$ brauchen nicht einmal im Raum $H^{1}(\Omega)^{3}$ liegen [11], [12], [14]. In [12] haben wir das Verhalten der Lösungen in Kegelspitzen untersucht. In der vorliegenden Arbeit wird diese Untersuchung weiter geführt. Wenn man nämlich z. B. Anwendungen auf die Methode der finiten Elemente betrachtet, ist das in [12] gewonnene Ergebnis nicht hinreichend. Sei $x_{0}$ ein singulärer Randpunkt und sei $U=\left\{x_{0}+r \omega \mid \omega \in G\right.$, $0<r<R\}, G \subset S=\{x|| x \mid=1\}$ eine Umgebung des Punktes $x_{0}$ in $\Omega$. Unter einigen Bedingungen an das sphärische Gebiet $G$ leitet man für die Lösungen $E$ in $U$ eine Zerlegung $E=S+W$ in einen singulären Teil $S$ und einen anderen $W \in H^{2}(U)^{3}$ her. Der singuläre Teil $S$ ist vom Typ

$$
S=\sum_{n=1}^{p} \alpha_{n}\left|x-x_{0}\right|_{n} F_{n}(\omega)
$$

Die Konstanten $\xi_{n}$ hängen nur von den Eigenwerten der Dirichletschen und Neumannschen Aufgabe für den Laplace---Beltramischen Operator auf $G$ ab. Die Felder $F_{n}$ stellt man mit Hilfe der Eigenfunktionen dieser Probleme dar. Die Konstanten $\alpha_{n}$ und das Feld $W$ erfüllen die Abschätzung

$$
\|W\|_{2, U}+\left(\sum_{n=1}^{p}\left|\alpha_{n}\right|^{2}\right)^{1 / 2} \leqq C\left(\|E\|+\|F\|+\|\operatorname{div} F\|_{1}\right) .
$$

Derartige Zerlegungen sind besonders wichtig, wenn man die Lösungen mit finiten Elementen in nichtglatten Gebieten zu approximieren versucht [15]. In [12] konnten wir diese Zerlegung nicht erreichen. Hier wird sie dadurch ermöglicht, daß wir mit einer Methode von Kondrat'ev [4] die Koeffizienten einer Entwicklung für die Lösung günstig zerlegen. Der ebene Fall mit einer Anwendung auf finite Elemente wurde in [13] behandelt.

\section{Entwicklung für die Lösung}

Wir betrachten das Verhalten der Lösung in der Nähe eines Punktes $x_{0} \in \Gamma$, wo der Rand nicht glatt ist. Wir wollen die Lösung in einer Umgebung von $x_{0}$ günstig entwickeln. Dafür fordert man, daß $x_{0}$ eine kegelförmige Umgebung in $\Omega$ besitzt. Sei $B\left(x_{0}, R\right)=\left\{x|| x-x_{0} \mid<R\right\}$. Man fordert (Vo 1): Es existieren ein Radius $R>0$ und ein sphärisches Gebiet $G \subset S$ mit $B\left(x_{0}, R\right) \cap \Omega=\left\{x_{0}+r \omega \mid \omega \in G\right.$, $0<r<R\}$. Unsere Betrachtungen werden besonders in solchen Fällen anwendbar sein, in denen das Gebiet $G$ glatt ist. Wir werden die nötigen Glattheitsvoraussetzungen über den Rand von $G$ in den folgenden Betrachtungen erst dann machen, wenn sie gebraucht werden. (In dieser Arbeit werden aber nicht Glattheisvoraus- 
setzungen größter Allgemeinheit angestrebt.) Im Falle eines stückweise glatten Gebietes $G$ könnte man einen Entwicklungssatz von Weck [16], [17] verwenden. Wir werden einen solchen Satz für allgemeinere sphärische Gebiete beweisen. Es sei bemerkt, daß wir somit auch solche Gebiete betrachten, für welche keine vollständige Existenztheorie existiert. Andererseits gibt es immer Lösungen (wähle $\omega=i)$. Wir sind hier nicht daran interessiert, die Existenztheorie in diese Richtung zu verallgemeinern. Außer (Vo 1) treten die folgenden Voraussetzungen auf (Vo 2): Das sphärische Gebiet $G$ kann mit Hilfe einer Karte dargestellt werden; o. B. d. A. sei $Q=\{\omega=(\theta, \varphi) \mid 0<\theta<\pi, 0<\varphi<2 \pi\}, u(\omega)=(\sin \theta \cos \varphi, \sin \theta \sin \varphi, \cos \theta)$ und sei $\bar{G} \subset u(Q)$. Im weiteren werden $u(\omega)$ und $\omega$ (wie schon früher) oft identifiziert. (Vo 3): $G_{u}=u^{-1}(G)$ hat die Segmenteneigenschaft. Man kann (Vo 2) gewissermaßen als eine technische Voraussetzung ansehen. Bedingung (Vo 3) ist wesentlicher. Sie garantiert genügend Eigenschaften für die Neumannsche Aufgabe des Laplace-Beltramischen Operators auf $G$. Sei $L^{2,2}=\left\{E=E_{\theta} \bar{e}_{\theta}+E_{\varphi} \bar{e}_{\varphi} \mid E_{\theta}\right.$, $\left.E_{\varphi} \in L^{2}=L^{2}(G)\right\}$ der Raum der quadratintegrablen Flächenfelder. Bezcichne $\mathscr{D}=C_{0}^{\infty}(G)$. Analog zum früheren Vorgehen definiert man eine Reihe der Teilräume von $L^{2,2}$ durch

$$
\begin{aligned}
& D=\left\{E \in L^{2,2} \mid \exists F_{1} \in L^{2}:\left(E \mid \nabla_{\omega} \Phi\right)=-\left(F_{1} \mid \Phi\right) \quad \forall \Phi \in \mathscr{D}\right\}, \\
& R=\left\{E \in L^{2,2} \mid \exists F_{2} \in L^{2}:\left(E \mid \nabla_{\omega} \times \Phi\right)=\left(F_{2} \mid \Phi\right) \quad \forall \Phi \in \mathscr{D}\right\} .
\end{aligned}
$$

Hier ist $\nabla_{\omega} \Phi=\partial_{\theta} \Phi \bar{e}_{\theta}+(\sin \theta)^{-1} \partial_{\varphi} \Phi \bar{e}_{\varphi}, \nabla_{\omega} \times \Phi=-\bar{e}_{r} \times \nabla_{\omega} \Phi$. Auf $D$ bzw. $R$ setzt man dann $\operatorname{div}_{\omega} E=\nabla_{\omega} \cdot E=F_{1}, \operatorname{rot}_{\omega} E=\nabla_{\omega} \times E=F_{2}$. Für differenzierbare Felder stimmen diese Definitionen mit den klassischen Formeln

$$
\begin{aligned}
\nabla_{\omega} \cdot E & =(\sin \theta)^{-1}\left[\partial_{\theta}\left(\sin \theta E_{\theta}\right)+\partial_{\varphi} E_{\varphi}\right], \\
\nabla_{\omega} \times E & =(\sin \theta)^{-1}\left[\partial_{\theta}\left(\sin \theta E_{\varphi}\right)-\partial_{\varphi} E_{\theta}\right]
\end{aligned}
$$

überein. Ferner setzt man $R_{0}=\left\{E \in R \mid \nabla_{\omega} \times E=0\right\}, D_{0}=\left\{E \in D \mid \nabla_{\omega} \cdot E=0\right\}$ und mit $H^{1}=H^{1}(G)$

$$
\begin{aligned}
& R^{0}=\left\{E \in R \mid\left(\nabla_{\omega} \times E \mid F\right)=\left(E \mid \nabla_{\omega} \times F\right) \quad \forall F \in H^{1}\right\}, \\
& D^{0}=\left\{E \in D \mid\left(\nabla_{\omega} \cdot E \mid F\right)=-\left(E \mid \nabla_{\omega} F\right) \quad \forall F \in H^{1}\right\} .
\end{aligned}
$$

Im folgenden bedeutet $\oplus$ die orthogonale Summe. Analog zu [16, Lemma 2] ergibt sich (mit $\left.H_{0}^{1}=H_{0}^{1}(G)\right)$

1.1. Lemma. Sei (Vo 2) erfüllt. Dann gilt

$$
\begin{aligned}
& L^{2,2}=D_{0} \oplus \overline{\nabla_{\omega} H_{0}^{1}}, \\
& L^{2,2}=R_{0} \oplus \overline{\nabla_{\omega} \times H_{0}^{1}} .
\end{aligned}
$$

Wir benutzen die Eigenfunktionen des Laplace-Beltramischen Operators mit der Dirichletschen und mit der Neumannschen Aufgabe auf $G$. Man führt die 
entsprechenden Operatoren $\Delta_{i}: D\left(\Delta_{i}\right) \subset L \rightarrow L, i=0,1 \mathrm{mit}$

$$
\begin{aligned}
& D\left(\Delta_{0}\right)=\left\{u \in H_{0}^{1} \mid \exists f_{1} \in L^{2}:\left(\nabla_{\omega} u \mid \nabla_{\omega} \varphi\right)=-\left(f_{0} \mid \varphi\right) \forall \varphi \in H_{0}^{1}\right\}, \\
& D\left(\Delta_{1}\right)=\left\{u \in H^{1} \mid \exists f_{2} \in L^{2}:\left(\nabla_{\omega} u \mid \nabla_{\omega} \varphi\right)=-\left(f_{1} \mid \varphi\right) \forall \varphi \in H^{1}\right\},
\end{aligned}
$$

$\Delta_{i} u:=f_{i}, u \in D\left(\Delta_{i}\right)$ ein. Die Operatoren $-\Delta_{i}$ sind selbstadjungiert und nichtnegativ. Unabhängig von $G$ besitzt $-\Delta_{0}$ eine unendliche Menge $\left\{\lambda_{n, 0}\right\}_{1}^{\infty}$ der nach der Vielfachheit gezählten Eigenwerte $0<\lambda_{1,0}<\lambda_{2,0} \leqq \ldots \rightarrow \infty$ die sich im Endlichen nirgends häufen. Sei entsprechend $\left\{\lambda_{n, 1}\right\}_{0}^{\infty}$ die Menge der Eigenwerte für $-\Delta_{1}$. Das oben gesagte gilt für $-\Delta_{1}$, falls das Gebiet $G_{u}$ die Segmenteneigenschaft besitzt, denn dann ist die Resolvente kompakt. Man beachte, daß der unterste Eigenwert $\left(\lambda_{1,0}>0\right.$ bzw. $\left.\lambda_{0,1}=0\right)$ für die beiden Operatoren einfach ist; für $-\Delta_{0}$ siehe [2], [3]. Seien $\left\{E_{n}\right\}_{1}^{\infty}$ bzw. $\left\{H_{n}\right\}_{0}^{\infty}$ die orthonormierten Eigenfunktionen von $-\Delta_{0}$ bzw. $-\Delta_{1}$. Wir definieren die folgenden Flächenfelder $\left(\omega_{n, i}:=\sqrt{\lambda_{n, i}}\right)$

$$
\begin{array}{ll}
E_{n}^{1}=i \omega_{n, 0}^{-1} \nabla_{\omega} E_{n}, & E_{n}^{2}=i \omega_{n, 0}^{-1} \bar{e}_{r} \times \nabla_{\omega} E_{n}, \\
H_{n}^{1}=i \omega_{n, 1}^{-1} \nabla_{\omega} H_{n}, & H_{n}^{2}=i \omega_{n, 1}^{-1} \bar{e}_{r} \times \nabla_{\omega} H_{n}
\end{array}
$$

für $n \geqq 1$. Die Systeme $\left\{E_{n}^{i}\right\}_{1}^{\infty},\left\{H_{n}^{i}\right\}_{1}^{\infty}$ sind orthonormiert. Weiterhin gilt $\left(E_{n}^{1} \mid H_{m}^{2}\right)=$ $\left(E_{n}^{2} \mid H_{m}^{1}\right)=0$ für alle $n, m$. Im Raum $H^{1}$ sei die $\|\cdot\|_{1}$-Norm $\|u\|_{1}=\left(\|u\|^{2}+\left\|\nabla_{\omega} u\right\|^{2}\right)^{1 / 2}$ festgelegt. Wie [7, Satz 6.7.2] zeigt man

1.2. Lemma. Seien (Vo 2), (Vo 3) erfüllt. Für $H \in H_{0}^{1}$ gilt $H=\sum_{n=1}^{\infty}\left(H \mid E_{n}\right) E_{n}$ in $H_{0}^{1}$, und für $H \in H^{1}$ gilt $H=\sum_{n=1}^{\infty}\left(H \mid H_{n}\right) H_{n}$ in $H^{1}$ (d.h. die Summen konvergieren in bezug auf die $\|\cdot\|_{1}-$ Norm).

Wir kürzen noch $\mathscr{H}=R^{0} \cap R_{0} \cap D_{0}, \mathscr{H}^{*}=D^{0} \cap D_{0} \cap R_{0}$ ab.

1.3. Satz. Die Voraussetzungen (Vo 2), (Vo 3) seien erfüllt. Dann sind die volgenden Systeme vollständig in den entsprechenden Räumen

$$
\begin{aligned}
& \left\{E_{n}^{1}\right\}_{1}^{\infty} \text { in } \overline{\nabla_{\omega} H_{0}^{1}}, \\
& \left\{E_{n}^{2}\right\}_{1}^{\infty} \text { in } \overline{\nabla_{\omega} \times H_{0}^{1}}, \\
& \left\{H_{n}^{2}\right\}_{1}^{\infty} \text { in } D_{0} \cap \mathscr{H}^{\perp}, \\
& \left\{H_{n}^{1}\right\}_{1}^{\infty} \text { in } R_{0} \cap\left(\mathscr{H}^{*}\right)^{\perp} .
\end{aligned}
$$

Beweis. Es ist leicht zu sehen, daß die betrachteten Felder zu den entsprechenden Räumen gehören. Vom Rest wird nur (iii) argumentiert. Sei $E \in D_{0} \cap \mathscr{H}^{\perp}$ mit $\left(E \mid H_{n}^{2}\right)=0, n \in N$. Weil die Eigenfunktion $H_{0}$ konstant ist, folgt $0=\left(E \mid \bar{e}_{r} \times \nabla_{\omega} H_{n}\right)=$ $\left(E \mid \nabla_{\omega} \times H_{n}\right)$ für alle $n \in N_{0}$. Nach Lemma 1.2 gilt $\left(E \mid \nabla_{\omega} \times H\right)=0, H \in H^{1}$. Also ist $E \in R^{0} \cap R_{0}$ und folglich $E \in \mathscr{H} \cap \mathscr{H}^{\perp}=\{0\}$.

Sei $x_{0} \in \Gamma$ derart, daß (Vo 1)-(Vo 3) erfüllt ist. O. B. d. A. wählt man $x_{0}=0$, und man setzt $U=U(R)=B(0, R) \cap \Omega$. Man betrachtet ein divergenzfreies Feld 
$E$, das der Gleichung (0.3) genügt. Gleichbedeutend ist dann $\left(\lambda=-\omega^{2}\right)$

$$
\nabla \times \nabla \times E+\lambda E=F, \quad E \in R^{0}(\Omega) \cap D_{0}(\Omega) .
$$

Von nun an setzen wir voraus, daß das sphärische Gebiet $G$ einfach zusammenhängend ist. Damit meinen wir, daß (Vo 4) $\operatorname{dim} \mathscr{H}=0$ gilt. Unter einigen schwachen Voraussetzungen läßt sich zeigen, daß diese Bedingung mit dem üblichen geometrischen Begriff übereinstimmt [9]. Die Forderung $\operatorname{dim} \mathscr{H}=0$ ist auch äquivalent mit $\operatorname{dim} \mathscr{H}^{*}=0$, denn $E \in \mathscr{H} \Leftrightarrow \bar{e}_{r} \times E \in \mathscr{H}^{*}$. Aus $E \in L^{2}(U)^{3}$ folgt, daß mit $E=E_{r} \bar{e}_{r}+E_{\theta} \bar{e}_{\theta}+E_{\varphi} \bar{e}_{\varphi}=E_{r} \bar{e}_{r}+E^{u}$ für fast alle $0<r<R$ die Relationen $E_{r} \in L^{2}$, $E^{u} \in L^{2,2}$ gelten. Wir setzen $E_{n}^{0}=E_{n} \bar{e}_{r}$. Nach Lemma 1.1, Satz 1.3 kann man in $L^{2}$ bzw. $L^{2,2}$ entwickeln

$$
\begin{aligned}
& E_{r}(r, \omega)=r^{-1} \sum_{n=1}^{\infty} a_{n}(r) E_{n}(\omega), \\
& E^{u}(r, \omega)=r^{-1} \sum_{n=1}^{\infty}\left(b_{n}(r) H_{n}^{2}(\omega)+c_{n}(r) E_{n}^{1}(\omega)\right) .
\end{aligned}
$$

Wegen $E \in L^{2}(U)^{3}$ ist die Entwicklung

$$
E=r^{-1} \sum_{n=1}^{\infty}\left(a_{n} E_{n}^{0}+b_{n} H_{n}^{2}+c_{n} E_{n}^{1}\right)
$$

richtig in $L^{2}(U)^{3}$, und man erhält

$$
\|E\|_{0, U(R)}^{2}=\sum_{n=1}^{\infty} \int_{0}^{R}\left(\left|a_{n}(r)\right|^{2}+\left|b_{n}(r)\right|^{2}+\left|c_{n}(r)\right|^{2}\right) d r .
$$

Für das Feld $\nabla \times E$ ergibt sich mit $H_{n}^{0}=H_{n} \bar{e}_{r}$ in $L^{2}(U)^{3}$

$$
\nabla \times E=r^{-1} \sum_{n=1}^{\infty}\left[-i \omega_{n, 1} r^{-1} b_{n} H_{n}^{0}-b_{n}^{\prime} H_{n}^{1}+\left(i \omega_{n, 0} r^{-1} a_{n}+c_{n}^{\prime}\right) E_{n}^{2}\right]
$$

mit

$$
\|\nabla \times E\|_{0, U(R)}^{2}=\sum_{n=1}^{\infty} \int_{0}^{R}\left(\lambda_{n, 1} r^{-2}\left|b_{n}\right|^{2}+\left|b_{n}^{\prime}\right|^{2}+\left|i \omega_{n, 0} r^{-1} a_{n}+c_{n}^{\prime}\right|^{2}\right) d r .
$$

Die Ableitungen in (1.4) sind distributional definiert. Die Darstellung (1.4) erhält man wie folgt. Man entwickelt

$$
E=r^{-1} \sum_{n=1}^{\infty}\left(A_{n} H_{n}^{0}+B_{n} E_{n}^{2}+C_{n} H_{n}^{1}\right)
$$

und benutzt die Umformung $(\nabla \times E \mid \Phi)=(E \mid \nabla \times \Phi)$ mit $\Phi=r^{-1} \varphi H_{n}^{0}, r^{-1} \varphi E_{n}^{2}$, $r^{-1} \varphi H_{n}^{1}, \varphi \in \mathscr{D}((0, R))$. Im weiteren setzen wir zuerst $\lambda=0$. Wenn $F$ die Entwicklung

$$
F=r^{-1} \sum_{n=1}^{\infty}\left(\alpha_{n} E_{n}^{0}+\beta_{n} H_{n}^{2}+\gamma_{n} E_{n}^{1}\right)
$$

in $L^{2}(U)^{3}$ besitzt, führt die Gleichung (1.1) $(\lambda=0)$ auf das folgende System von 
gewöhnlichen Differentialgleichungen

$$
\begin{aligned}
\lambda_{n, 0} r^{-1} a_{n}-i \omega_{n, 0} c_{n}^{\prime} & =r \alpha_{n}, \\
b_{n}^{\prime \prime}-r^{-2} \lambda_{n, 1} b_{n} & =-\beta_{n}, \\
i \omega_{n, 0}\left(r^{-1} a_{n}\right)^{\prime}+c_{n}^{\prime \prime} & =-\gamma_{n} .
\end{aligned}
$$

Aus der Bedingung $\operatorname{div} E=0$ folgt

$$
\left(r a_{n}\right)^{\prime}-i \omega_{n, 0} c_{n}=0 .
$$

Auch in (1.7), (1.8) sind die Ableitungen im schwachen Sinn definiert.

\section{Verhalten der Koeffizienten}

Ausgehend von dem System (1.7), (1.8) und von den Bedingungen $E$, $\nabla \times E \in L^{2}(U)^{3}$ untersuchen wir in diesem Abschnitt die Asymptotik der Koeffizienten $a_{n}, b_{n}, c_{n}$ mit $r \rightarrow 0$. Zur Diskussion des Verhaltens Verwenden wir eine Technik von Kondrat'ev [4]. Besonders das folgende Lemma ist eine solche verschärfte Version von Theorem 1.2 [4], wo die Abhängigkeit vom Parameter $\lambda_{n}$ berücksichtigt wird. Die Argumente werden nur kurz gegeben. Aus (1.7i), (1.8) ergibt sich

$$
a_{n}^{\prime \prime}+2 r^{-1} a_{n}^{\prime}-\lambda_{n, 0} r^{-2} a_{n}=-\alpha_{n} .
$$

Die Gleichungen (1.7ii), (2.1) sind allgemeinerer Form

$$
u^{\prime \prime}+\beta_{1} r^{-1} u^{\prime}-\lambda_{n} r^{-2} u=-f
$$

mit $\beta_{1} \in \boldsymbol{R}$. Die Gleichung (2.2) gelte in $\boldsymbol{R}_{+}=\{r \mid r>0\}$. Führt man in (2.2) eine neue Variable $\tau=\ln r^{-1}$ ein, und setzt man $v(\tau)=u\left(e^{-\tau}\right), g(\tau)=f\left(r^{-\tau}\right)$, dann folgt

$$
v^{\prime \prime}+\left(1-\beta_{1}\right) v^{\prime}-\lambda_{n} v=-e^{-2 \tau} g(\tau)=:-G(\tau) .
$$

Für die Fourier-Transformierte $\tilde{v}(\lambda)=\int_{-\infty}^{\infty} e^{-i \lambda \tau} v(\tau) \mathrm{d} \tau$ gilt

$$
P_{n}(\lambda) \tilde{v}(\lambda)=\tilde{G}(\lambda)
$$

mit $P_{n}(\lambda)=\lambda^{2}-i\left(1-\beta_{1}\right) \lambda+\lambda_{n}$. Unsere einschränkenden Bedingungen für $u$ und $f$ werden derart sein, daß die obige formale Rechnung berechtigt ist. Man definiert die folgenden gewichteten Normen und Räume

$$
\|u\|_{k, \alpha}^{2}=\sum_{v=0}^{k}\left\|r^{\alpha / 2-(k-v)} u^{(v)}\right\|^{2}=\sum_{\nu=0}^{k} \int_{0}^{\infty} r^{\alpha-2(k-v)}\left|\left(\frac{d}{d r}\right)^{v} u(r)\right|^{2} d r
$$

und $W_{\alpha}^{k}=\left\{u \in H^{k \text {, loc }}\left(\boldsymbol{R}_{+}\right) \mid\|u\|_{k, \alpha}<\infty\right\}$. Sei ferner $\left(\lambda_{n}\right)_{1}^{\infty}, \lambda_{n} \rightarrow \infty$ eine Folge von reellen Zahlen, die keinen endlichen Häufungspunkt besitzt. Verknüpft mit der Folge $\left(\lambda_{n}\right)_{1}^{\infty}$ erklärt man

$$
\|u\|_{k, \alpha,\left(\lambda_{n}\right)}^{2}=\sum_{\nu=0}^{k}\left(1+\left|\lambda_{n}\right|\right)^{k-v}\left\|r^{\alpha / 2-(k-v)} u^{(v)}\right\|^{2} .
$$


2.1. Lemma. Sei $h_{1}=\left(2 k_{1}-\alpha_{1}+3\right) / 2, h=(2 k-\alpha-1) / 2$ und sei $h_{1}>h$. Wenn $P_{n}(\lambda) \neq 0$ für alle $n$ und alle $\lambda$ mit $\operatorname{Im} \lambda=h_{1}$ gilt, ergibt sich für alle Lösungen $u \in W_{\alpha}^{k}$ der Gleichung (2.2) mit $f \in W_{\alpha_{1}}^{k_{1}}$ die Zerlegung

$$
u=w+\left.i \sum_{h<\operatorname{Im} \lambda<h_{1}} \operatorname{Res}\left(\frac{\tilde{G}(\lambda)}{P_{n}(\lambda)} e^{i \tau \lambda}\right)\right|_{\tau=\ln r^{-1}}
$$

mit $w \in W_{\alpha_{1}}^{k_{1}+2}$. Die Funktion $w$ erfüllt die Abschätzung

$$
\|w\|_{k_{1}+2, \alpha_{1},\left(\lambda_{n}\right)} \leqq C\|f\|_{k_{1}, \alpha_{1},\left(\lambda_{n}\right)} .
$$

Beweis. Für $h \leqq h^{\prime} \leqq h_{1}$ rechnet man

$$
\begin{aligned}
\int_{-\infty}^{\infty}\left|e^{h^{\prime} \tau} G(\tau)\right|^{2} d \tau & =\int_{0}^{\infty} r^{3-2 h^{\prime}}|f(r)|^{2} d r \\
& \leqq C\left(\|u\|_{k, \alpha}^{2}+\left\|f_{k_{1}, \alpha_{1}}^{2}\right\|\right)<\infty .
\end{aligned}
$$

Demzufolge ist $\lambda \mapsto \tilde{G}(\lambda)$ analytisch im Segment $h<\operatorname{Im} \lambda<h_{1}$, und man kann invertieren

$$
\begin{aligned}
v(\tau) & =\frac{1}{2 \pi} \int_{-\infty+i h}^{\infty+i h} e^{i \tau \lambda} \tilde{v}(\lambda) d \lambda \\
& =w_{1}(\tau)+i \sum_{h<\operatorname{Im} \lambda<h_{1}} \operatorname{Res} \frac{\widetilde{G}(\lambda)}{P_{n}(\lambda)} e^{i \tau \lambda}
\end{aligned}
$$

mit

$$
w_{1}(\tau)=\frac{1}{2 \pi} \int_{-\infty+i h_{1}}^{\infty+i h_{1}} e^{i \tau \lambda} \tilde{v}(\lambda) d \lambda .
$$

Weil das Polynom $P_{n}(\lambda)$ für $\operatorname{Im} \lambda=h_{1}$ keine Nullstellen besitzt, folgert man aus (2.4) die Ungleichung

$$
|\tilde{v}(\lambda)| \leqq C\left(1+\left|\lambda_{n}\right|\right)^{p / 2-1}(1+|\lambda|)^{-p}|\widetilde{G}(\lambda)|
$$

mit $p=0,1,2, \operatorname{Im} \lambda=h_{1}$. Mit Hilfe von (2.8) rechnet man für $w(r)=w_{1}(\ln (1 / r))$

$$
\begin{aligned}
\|w\|_{k_{1}+2, \alpha_{1},\left(\lambda_{n}\right)}^{2} & \leqq C \sum_{v=0}^{k_{1}+2}\left(1+\left|\lambda_{n}\right|\right)^{k_{1}+2-v} \int_{-\infty}^{\infty} e^{2 h_{1} \tau}\left|w_{1}^{(v)}(\tau)\right|^{2} d \tau \\
& =C \sum_{v=0}^{k_{1}+2}\left(1+\left|\lambda_{n}\right|\right)^{k_{1}+2-v} \int_{-\infty+i h_{1}}^{\infty+i h_{1}}|\lambda|^{2 v}|\tilde{v}(\lambda)|^{2} d \lambda \\
& \leqq C \sum_{v=0}^{k_{1}}\left(1+\left|\lambda_{n}\right|\right)^{k_{1}-v} \int_{-\infty+i h_{1}}^{\infty+i h_{1}}|\lambda|^{2 v}|\tilde{G}(\lambda)|^{2} d \lambda \leqq C\|f\|_{k_{1}, \alpha_{1}}^{2} .
\end{aligned}
$$

Die Formel (2.7) führt auf (2.5). 
Sei $0<R_{0}<R$. Es wird abgekürzt

$$
\begin{aligned}
& L_{n}^{0} u=-\left(u^{\prime \prime}+2 r^{-1} u^{\prime}-\lambda_{n, 0} r^{-2} u\right), \\
& L_{n}^{1} u=-\left(u^{\prime \prime}-\lambda_{n, 1} r^{-2} u\right) .
\end{aligned}
$$

Im folgenden bedeutet $\eta$ eine beliebige Funktion $\eta \in \mathscr{D}((-\infty, R))$, die in einer Umgebung des Punktes $r=0$ identisch Eins ist. Die Bedeutung der Bezeichnungen $W_{\alpha}^{k}\left(R_{0}\right)=W_{\alpha}^{k}\left(\left(0, R_{0}\right)\right)$ usw. ist klar.

2.2. Satz. Sei $\lambda_{n, 0} \neq 15 / 4$ und sei $\lambda_{n, 1} \neq 3 / 4$ für alle $n$. In $\left(0, R_{0}\right)$ hat man die Zerlegungen

$$
\begin{aligned}
& a_{n}(r)=a_{n}^{0}(r)+\delta_{n}^{1} r^{v_{n},}, \\
& c_{n}(r)=c_{n}^{0}(r)+\delta_{n}^{2} r^{v_{n},}, \\
& n_{n}(r)=b_{n}^{0}(r)+\delta_{n}^{3} r^{x_{n},},
\end{aligned}
$$

mit $v_{n}=2^{-1}\left(-1+\left(1+4 \lambda_{n, 0}\right)^{1 / 2}\right), \varkappa_{n}=2^{-1}\left(1+\left(1+4 \lambda_{n, 1}\right)^{1 / 2}\right)$ und mit Konstanten $\delta_{n}^{i}$ derart, daß $\delta_{n}^{1}=\delta_{n}^{2}=0$ für $\lambda_{n, 0}>15 / 4$ und $\delta_{n}^{3}=0$ für $\lambda_{n, 1}>3 / 4$ ist. Für $\lambda_{n, 0}<15 / 4$ bzw. $\lambda_{n, 1}<3 / 4$ (2.10iii) gilt

$$
\begin{aligned}
& \delta_{n}^{1}=\left(1+4 \lambda_{n, 0}\right)^{-1 / 2} \int_{0}^{R} r^{-v_{n}+1} L_{n}^{0}\left(\eta a_{n}\right) d r \\
& \delta_{n}^{2}=\left(i \omega_{n, 0}\right)^{-1} \delta_{n}^{1}\left(1+v_{n}\right),
\end{aligned}
$$

$$
\delta_{n}^{3}=\left(1+4 \lambda_{n, 1}\right)^{-1 / 2} \int_{0}^{R} r^{-x_{n}+1} L_{n}^{1}\left(\eta b_{n}\right) d r
$$

mit den Abschätzungen

$$
\begin{aligned}
& \left|\delta_{n}^{1}\right|+\left|\delta_{n}^{2}\right| \leqq C\left(\left\|\alpha_{n}\right\|(R)+\left\|a_{n}\right\|(R)+\left\|c_{n}\right\|(R)\right)^{1)}, \\
& \left|\delta_{n}^{3}\right| \leqq C\left(\left\|\beta_{n}\right\|(R)+\left\|b_{n}\right\|_{1,\left(R_{0}, R\right)}\right) .
\end{aligned}
$$

Ferner gilt $a_{n}^{0}, b_{n}^{0}, c_{n}^{0} \in W_{0}^{2}\left(R_{0}^{2}\right)$ mit

$$
\begin{aligned}
& \left\|a_{n}^{0}\right\|_{2,0,\left(\lambda_{n, 0}\right)}\left(R_{0}\right) \leqq C\left(\left\|a_{n}\right\|(R)+\left\|a_{n}\right\|(R)+\left\|c_{n}\right\|(R)\right), \\
& \left\|c_{n}^{0}\right\|_{2,0,\left(\lambda_{n, 0}\right)}\left(R_{0}\right) \leqq C\left(\left\|\alpha_{n}\right\|(R)+\left\|\gamma_{n}\right\|(R)+\left\|a_{n}\right\|(R)+\left\|c_{n}\right\|(R)\right), \\
& \left\|b_{n}^{0}\right\|_{2,0,\left(\lambda_{n, 1}\right)}\left(R_{0}\right) \leqq C\left(\left\|\beta_{n}\right\|(R)+\left\|b_{n}\right\|_{1,\left(R_{0}, R\right)}\right) .
\end{aligned}
$$

Beweis. Die Bedingungen $E, F \in L^{2}(U)^{3}$, div $E=0$ implizieren $\eta a_{n} \in W_{4}^{2}$, $L_{n}^{0}\left(\eta a_{n}\right) \in W_{0}^{0}=L^{2}\left(\boldsymbol{R}_{+}\right)$. Wählt man in Lemma $2.1 \alpha=4, \alpha_{1}=0, k=2, k_{1}=0$, dann erhält man

$$
\eta(r) a(r, \eta)=a_{n}^{0}(r, \eta)+\delta_{n}^{1} r^{v_{n}}
$$

$$
\text { 1) }\left\|\alpha_{n}\right\|(R)=\left\|\alpha_{n}\right\|_{0,[0, R]} \text {. }
$$


2.1. Lemma. Sei $h_{1}=\left(2 k_{1}-\alpha_{1}+3\right) / 2, h=(2 k-\alpha-1) / 2$ und sei $h_{1}>h$. Wenn $P_{n}(\lambda) \neq 0$ für alle $n$ und alle $\lambda$ mit $\operatorname{Im} \lambda=h_{1}$ gilt, ergibt sich für alle Löstingen $u \in W_{\alpha}^{k}$ der Gleichung (2.2) mit $f \in W_{\alpha_{1}}^{k_{1}}$ die Zerlegung

$$
u=w+\left.i \sum_{h<\operatorname{Im} \lambda<h_{1}} \operatorname{Res}\left(\frac{\widetilde{G}(\lambda)}{P_{n}(\lambda)} e^{i \tau \lambda}\right)\right|_{\tau=\ln r^{-1}}
$$

mit $w \in W_{\alpha_{1}}^{k_{1}+2}$. Die Funktion $w$ erfüllt die Abschätzung

$$
\|w\|_{k_{1}+2, \alpha_{1},\left(\lambda_{n}\right)} \leqq C\|f\|_{k_{1}, \alpha_{1},\left(\lambda_{n}\right)} .
$$

Beweis. Für $h \leqq h^{\prime} \leqq h_{1}$ rechnet man

$$
\begin{aligned}
\int_{-\infty}^{\infty}\left|e^{h^{\prime} \tau} G(\tau)\right|^{2} d \tau & =\int_{0}^{\infty} r^{3-2 h^{\prime}}|f(r)|^{2} d r \\
& \leqq C\left(\|u\|_{k, \alpha}^{2}+\left\|f_{k_{1}, \alpha_{1}}^{2}\right\|\right)<\infty
\end{aligned}
$$

Demzufolge ist $\lambda \mapsto \tilde{G}(\lambda)$ analytisch im Segment $h<\operatorname{Im} \lambda<h_{1}$, und man kann invertieren

$$
\begin{aligned}
v(\tau) & =\frac{1}{2 \pi} \int_{-\infty+i h}^{\infty+i h} e^{i \tau \lambda} \tilde{v}(\lambda) d \lambda \\
& =w_{1}(\tau)+i \sum_{h<\operatorname{Im} \lambda<h_{1}} \operatorname{Res} \frac{\widetilde{G}(\lambda)}{P_{n}(\lambda)} e^{i \tau \lambda}
\end{aligned}
$$

mit

$$
w_{1}(\tau)=\frac{1}{2 \pi} \int_{-\infty+i h_{1}}^{\infty+i h_{1}} e^{i \tau \lambda} \tilde{v}(\lambda) d \lambda .
$$

Weil das Polynom $P_{n}(\lambda)$ für $\operatorname{Im} \lambda=h_{1}$ keine Nullstellen besitzt, folgert man aus (2.4) die Ungleichung

$$
|\tilde{v}(\lambda)| \leqq C\left(1+\left|\lambda_{n}\right|\right)^{p / 2-1}(1+|\lambda|)^{-p}|\widetilde{G}(\lambda)|
$$

mit $p=0,1,2, \operatorname{Im} \lambda=h_{1}$. Mit Hilfe von (2.8) rechnet man für $w(r)=w_{1}(\ln (1 / r))$

$$
\begin{aligned}
\|w\|_{k_{1}+2, \alpha_{1},\left(\lambda_{n}\right)}^{2} & \leqq C \sum_{v=0}^{k_{1}+2}\left(1+\left|\lambda_{n}\right|\right)^{k_{1}+2-v} \int_{-\infty}^{\infty} e^{2 h_{1} \tau}\left|w_{1}^{(v)}(\tau)\right|^{2} d \tau \\
& =C \sum_{v=0}^{k_{1}+2}\left(1+\left|\lambda_{n}\right|\right)^{k_{1}+2-v} \int_{-\infty+i h_{1}}^{\infty+i h_{1}}|\lambda|^{2 v}|\tilde{v}(\lambda)|^{2} d \lambda \\
& \leqq C \sum_{v=0}^{k_{1}}\left(1+\left|\lambda_{n}\right|\right)^{k_{1}-v} \int_{-\infty+i h_{1}}^{\infty+i h_{1}}|\lambda|^{2 v}|\tilde{G}(\lambda)|^{2} d \lambda \leqq C\|f\|_{k_{1}, \alpha_{1}}^{2} .
\end{aligned}
$$

Die Formel (2.7) führt auf (2.5). 
Sei $0<R_{0}<R$. Es wird abgekürzt

$$
\begin{aligned}
& L_{n}^{0} u=-\left(u^{\prime \prime}+2 r^{-1} u^{\prime}-\lambda_{n, 0} r^{-2} u\right), \\
& L_{n}^{1} u=-\left(u^{\prime \prime}-\lambda_{n, 1} r^{-2} u\right) .
\end{aligned}
$$

Im folgenden bedeutet $\eta$ eine beliebige Funktion $\eta \in \mathscr{D}((-\infty, R))$, die in einer Umgebung des Punktes $r=0$ identisch Eins ist. Die Bedeutung der Bezeichnungen $W_{\alpha}^{k}\left(R_{0}\right)=W_{\alpha}^{k}\left(\left(0, R_{0}\right)\right)$ usw. ist klar.

2.2. Satz. Sei $\lambda_{n, 0} \neq 15 / 4$ und sei $\lambda_{n, 1} \neq 3 / 4$ für alle $n$. In $\left(0, R_{0}\right)$ hat man die Zerlegungen

$$
\begin{aligned}
& a_{n}(r)=a_{n}^{0}(r)+\delta_{n}^{1} r^{v_{n},} \\
& c_{n}(r)=c_{n}^{0}(r)+\delta_{n}^{2} r^{v_{n},}, \\
& n_{n}(r)=b_{n}^{0}(r)+\delta_{n}^{3} r^{x_{n},}
\end{aligned}
$$

mit $v_{n}=2^{-1}\left(-1+\left(1+4 \lambda_{n, 0}\right)^{1 / 2}\right), \varkappa_{n}=2^{-1}\left(1+\left(1+4 \lambda_{n, 1}\right)^{1 / 2}\right)$ und mit Konstanten $\delta_{n}^{i}$ derart, daß $\delta_{n}^{1}=\delta_{n}^{2}=0$ für $\lambda_{n, 0}>15 / 4$ und $\delta_{n}^{3}=0$ für $\lambda_{n, 1}>3 / 4$ ist. Für $\lambda_{n, 0}<15 / 4$ bzw. $\lambda_{n, 1}<3 / 4$ (2.10iii) gilt

$$
\begin{aligned}
& \delta_{n}^{1}=\left(1+4 \lambda_{n, 0}\right)^{-1 / 2} \int_{0}^{R} r^{-v_{n}+1} L_{n}^{0}\left(\eta a_{n}\right) d r \\
& \delta_{n}^{2}=\left(i \omega_{n, 0}\right)^{-1} \delta_{n}^{1}\left(1+v_{n}\right),
\end{aligned}
$$

$$
\delta_{n}^{3}=\left(1+4 \lambda_{n, 1}\right)^{-1 / 2} \int_{0}^{R} r^{-x_{n}+1} L_{n}^{1}\left(\eta b_{n}\right) d r
$$

mit den Abschätzungen

$$
\begin{aligned}
& \left|\delta_{n}^{1}\right|+\left|\delta_{n}^{2}\right| \leqq C\left(\left\|\alpha_{n}\right\|(R)+\left\|a_{n}\right\|(R)+\left\|c_{n}\right\|(R)\right)^{1)}, \\
& \left|\delta_{n}^{3}\right| \leqq C\left(\left\|\beta_{n}\right\|(R)+\left\|b_{n}\right\|_{1,\left(R_{0}, R\right)}\right) .
\end{aligned}
$$

Ferner gilt $a_{n}^{0}, b_{n}^{0}, c_{n}^{0} \in W_{0}^{2}\left(R_{0}^{2}\right)$ mit

$$
\begin{aligned}
& \left\|a_{n}^{0}\right\|_{2,0,\left(\lambda_{n, 0}\right)}\left(R_{0}\right) \leqq C\left(\left\|a_{n}\right\|(R)+\left\|a_{n}\right\|(R)+\left\|c_{n}\right\|(R)\right), \\
& \left\|c_{n}^{0}\right\|_{2,0,\left(\lambda_{n, 0}\right)}\left(R_{0}\right) \leqq C\left(\left\|\alpha_{n}\right\|(R)+\left\|\gamma_{n}\right\|(R)+\left\|a_{n}\right\|(R)+\left\|c_{n}\right\|(R)\right), \\
& \left\|b_{n}^{0}\right\|_{2,0,\left(\lambda_{n, 1}\right)}\left(R_{0}\right) \leqq C\left(\left\|\beta_{n}\right\|(R)+\left\|b_{n}\right\|_{1,\left(R_{0}, R\right)}\right) .
\end{aligned}
$$

Beweis. Die Bedingungen $E, F \in L^{2}(U)^{3}$, div $E=0$ implizieren $\eta a_{n} \in W_{4}^{2}$, $L_{n}^{0}\left(\eta a_{n}\right) \in W_{0}^{0}=L^{2}\left(\boldsymbol{R}_{+}\right)$. Wählt man in Lemma $2.1 \alpha=4, \alpha_{1}=0, k=2, k_{1}=0$, dann erhält man

$$
\eta(r) a(r, \eta)=a_{n}^{0}(r, \eta)+\delta_{n}^{1} r^{v_{n}}
$$

$$
\text { 1) }\left\|\alpha_{n}\right\|(R)=\left\|\alpha_{n}\right\|_{0,[0, R]} \text {. }
$$


mit $\delta_{n}^{1}=0$ für $\lambda_{n, 0}>15 / 4$ und mit

$$
\begin{aligned}
\delta_{n}^{1} & =i \tilde{G}\left(i v_{n}\right) \operatorname{Res}_{\lambda=i v_{n}} P_{n}(\lambda)^{-1} \\
& =\left(1+4 \lambda_{n, 0}\right)^{-1 / 2} \int_{0}^{R} r^{-v_{n}+1} L_{n}^{0}\left(\eta a_{n}\right) d r
\end{aligned}
$$

für $\lambda_{n, 0}<15 / 4$. Man wähle $R_{0}<R_{1}<R$ und $\eta_{0} \in \mathscr{D}(\boldsymbol{R})$ mit $\eta_{0}(r)=1,0 \leqq r \leqq R_{0}$, $\eta_{0}(r)=0, r \geqq R_{1}$. Ferner sei $a_{n}^{0}(r)=a_{n}^{0}\left(r, \eta_{0}\right)$. Nach (2.6), (1.8) rechnet man

$$
\begin{gathered}
\left\|a_{n}^{0}\right\|_{2,0,\left(\lambda_{n, 0}\right)}\left(R_{0}\right) \leqq C\left\|L_{n}^{0}\left(\eta_{0} a_{n}\right)\right\|\left(R_{1}\right) \\
\leqq C\left(\left\|\alpha_{n}\right\|\left(R_{1}\right)+\left\|a_{n}\right\|_{1,\left(R_{0}, R_{1}\right)}\right) \\
\leqq C\left(\left\|\alpha_{n}\right\|\left(R_{1}\right)+\left\|a_{n}\right\|\left(R_{1}\right)+\omega_{n, 0}\left\|c_{n}\right\|\left(R_{0}, R_{1}\right)\right) .
\end{gathered}
$$

Für (2.12i) genügt es folglich $\lambda_{n, 0}>15 / 4 \mathrm{zu}$ betrachten. Dann gilt $a_{n}=a_{n}^{0}$ in $\left(0, R_{0}\right)$, und demzufolge

$$
\left\|a_{n}\right\|_{2,0,\left(\lambda_{n, 0}\right)}\left(R_{0}\right) \leqq C\left(\left\|\alpha_{n}\right\|\left(R_{1}\right)+\left\|a_{n}\right\|_{1,\left(R_{0}, R_{1}\right)}\right) .
$$

Ähnlich hat man dann

$$
\left\|a_{n}\right\|_{2,0,\left(\lambda_{n, 0}\right)}\left(R_{1}\right) \leqq C\left(\left\|\alpha_{n}\right\|(R)+\left\|a_{n}\right\|_{1,\left(R_{1}, R\right)}\right) .
$$

Daraus schließt man

$$
\begin{aligned}
\left\|a_{n}\right\|_{1,\left(R_{0}, R_{1}\right)} & \leqq C \lambda_{n, 0}^{-1 / 2}\left\|a_{n}\right\|_{2,0,\left(\lambda_{n}, 0\right)}\left(R_{1}\right) \\
& \leqq C\left(\left\|\alpha_{n}\right\|(R)+\left\|a_{n}\right\|(R)+\left\|c_{n}\right\|(R)\right) .
\end{aligned}
$$

Die Abschätzung (2.12i) folgt aus (2.13), (2.15). Der Operator $r^{2} L_{n}^{0}$ ist symmetrisch, und es gilt $L_{n}^{0}\left(r^{-v_{n}-1}\right)=0$. Das impliziert, daß das Integral in (2.10i) von $\eta$ unabhängig ist. Nach (1.8), (2.9i) gilt für die Koeffizienten $c_{n}(r)$ (2.9ii) mit $c_{n}^{0}(r)=-i \omega_{n, 0}^{-1}\left(r a_{n}^{0}\right)^{\prime}$. Die Abschätzung (2.12ii) beweist man mit Hilfe von (2.7iii), (2.12i). Die Ungleichung (2.12iii) ist nun ersichtlich. Die Ungleichungen (2.11) sieht man leicht.

\section{Verhalten der Lösung}

Wir diskutieren die $H^{2}\left(U\left(R_{0}\right)\right)^{3}$-Regularität der Lösung. Man setze $\bar{e}^{1}=\bar{e}_{r}$, $\bar{e}^{2}=\bar{e}_{\theta}, \bar{e}^{3}=\bar{e}_{\varphi}$. Sei $E=\sum_{j=1}^{3} E^{j} \bar{e}^{j}$ in $U(R)$. Für die Norm $\|\cdot\|_{2, U(R)}$, definiert durch

$$
\|E\|_{2, U(R)}^{2}=\sum_{i=1}^{3}\left\|E_{i}\right\|_{2, U(R)}^{2}
$$


wo $E_{i}=\sum_{j=1}^{3} E^{j}\left(\bar{e}^{j} \cdot \bar{e}^{i}\right)$ die kartesischen Komponenten sind, errechnet man die Schranke

$$
\|E\|_{2, U(R)}^{2} \leqq C \sum_{j=1}^{3} \int_{0}^{R}\left(\sum_{\nu=0}^{2} r^{2-2 v}\left\|\left(\frac{d}{d r}\right)^{2-v} E^{j}\right\|_{v}^{2}\right) d r
$$

für $E \in H^{2, \operatorname{loc}}(U(R))^{3}$. Hier wurde $\|u\|_{v}=\|u(\omega)\|_{v, G_{u}}$ gesetzt. Für die Eigenlösungen sind die Abschätzungen $\left\|E_{n}\right\|_{v}^{2} \leqq C \lambda_{n, 0}^{v},\left\|H_{n}\right\|_{v}^{2} \leqq C \lambda_{n, 1}^{v}, 0 \leqq v \leqq 1, n \geqq 1$ unabhängig vom Gebiet $G$ richtig. Um die $H^{2}\left(U\left(R_{0}\right)\right)^{3}$-Abschätzung zu gewinnen machen wir stärkere Annahmen an die Regularität der Eigenlösungen (Vo 5):

$$
\begin{array}{ll}
\left\|E_{n}\right\|_{v}^{2} \leqq C \lambda_{n, 0}^{v}, & 0 \leqq v \leqq 3, \\
\left\|H_{n}\right\|_{n}^{2} \leqq C \lambda_{n, 1}^{v}, & 0 \leqq v \leqq 3, n \geqq 1 .
\end{array}
$$

Falls das sphärische Gebiet $G$ glatt ist, sind diese Voraussetzungen erfüllt. Sei nun

$$
S_{N}=\frac{1}{r} \sum_{n=1}^{N}\left(a_{n} E_{n}^{0}+b_{n} H_{n}^{2}+c_{n} E_{n}^{1}\right)
$$

Nach (3.1) ergibt sich unter (Vo 5)

$$
\begin{gathered}
\left\|S_{N}\right\|_{2, U(R)}^{2} \\
\leqq C \sum_{n=1}^{N}\left(\left\|a_{n}\right\|_{2,0,\left(\lambda_{n, 0}\right)}^{2}(R)+\left\|b_{n}\right\|_{2,0,\left(\lambda_{n, 1}\right)}^{2}(R)+\left\|c_{n}\right\|_{2,0,\left(\lambda_{n, 0}\right)}^{2}(R)\right) .
\end{gathered}
$$

Man betrachtet nun die Gleichung (1.1) mit einer allgemeinen Konstante $\lambda \in \boldsymbol{C}$. Falls das Feld $E$ bzw. $F$ die Entwicklung (1.2) mit den Koeffizienten $a_{n}(r, i)$, $b_{n}(r, \lambda), c_{n}(r, \lambda)$ bzw. $\alpha_{n}(r), \beta_{n}(r), \gamma_{n}(r)$ besitzt, erfüllen die Koeffizienten $a_{n}(r, \lambda)$, $b_{n}(r, \lambda), c_{n}(r, \lambda)$ ein modifiziertes System (1.7), (1.8), wo anstelle $\alpha_{n}, \beta_{n}, \gamma_{n}$ die Funktionen $\alpha_{n}^{*}=\alpha_{n}-\lambda a_{n}, \beta_{n}^{*}=\beta_{n}-\lambda b_{n}, \gamma_{n}^{*}=\gamma_{n}-\lambda c_{n}$ auftreten. Ähnlich tritt in Satz 2.2 die Abhängigkeit von $\lambda$ wie $\delta_{n}^{i}(\lambda)$ usw. auf.

Man setzt $D_{0}(\Omega)=\{E \in D(\Omega) \mid \operatorname{div} E=0\}$.

3.1. Satz. Sei $\lambda \in C$ und sei $E \in R^{0}(\Omega) \cap D_{0}(\Omega)$ eine Lösung der Aufgabe (1.1) mit $F \in D_{0}(\Omega)$. Ferner seien die Voraussetzungen (Vo 1)-(Vo 5) erfüllt und sei $\lambda_{n, 0} \neq 15 / 4, \lambda_{n, 1} \neq 3 / 4$ für alle $n$. Das Feld $E$ besitzt dann in $L^{2}\left(U\left(R_{0}\right)\right)^{3}$ die Darstellung

$$
\begin{aligned}
E= & \sum_{\lambda_{n, 0}<15 / 4} r^{-3 / 2+\left(1+4 \lambda_{n, 0}\right)^{1 / 2} / 2}\left(\delta_{n}^{2}\left(\lambda_{i}\right) E_{n}^{0}+\delta_{n}^{2}\left(\lambda_{i}\right) E_{n}^{1}\right) \\
& +\sum_{\lambda_{n, 1}<3 / 4} r^{-1 / 2+\left(1+4 \lambda_{n, 1}\right)^{1 / 2} / 2} \delta_{n}^{3}(\lambda) H_{n}^{2}+W_{i}
\end{aligned}
$$

mit $W_{\lambda} \in H^{2}\left(U\left(R_{0}\right)\right)^{3}$ derart, daß die Ungleichung

$$
\left\|W_{\lambda}\right\|_{2, U\left(R_{0}\right)} \leqq C(\lambda)(\|E\|+\|F\|)
$$


richtig ist. Für das Feld $W_{\lambda}$ gilt die in $H^{2}\left(U\left(R_{0}\right)\right)^{3}$ konvergente Entwicklung

$$
W_{\lambda}=\sum_{n=1}^{\infty} \frac{1}{r}\left(a_{n}^{0}(r, \lambda) E_{n}^{0}+b_{n}^{0}(r, \lambda) H_{n}^{2}+c_{n}^{0}(r, \lambda) E_{n}^{1}\right)
$$

Die Konstanten $\delta_{n}^{i}(\lambda)$ können mit

$$
\begin{gathered}
\sum_{\lambda_{n, 0}<15 / 4}\left(\left|\delta_{n}^{1}(\lambda)\right|^{2}+\left|\delta_{n}^{2}(\lambda)\right|^{2}\right)+\sum_{\lambda_{n}, 1<3 / 4}\left|\delta_{n}^{3}(\lambda)\right|^{2} \\
\leqq C(\lambda)\left(\|E\|^{2}+\|F\|^{2}\right)
\end{gathered}
$$

abgeschätzt werden.

Beweis. Jedenfalls ist (3.5) richtig in dem Sinn, daß $W_{\lambda}$ die Entwicklung (3.7) in $L^{2}\left(U\left(R_{\jmath}\right)\right)^{3}$ hat. Sei

$$
S_{N}^{0}=\sum_{n=1}^{N} \frac{1}{r}\left(a_{n}^{0}(r, \lambda) E_{n}^{0}+b_{n}^{0}(r, \lambda) H_{n}^{2}+c_{n}^{0}(r, \lambda) E_{n}^{1}\right) .
$$

Für $M>N$ ergibt sich nach (3.4), (2.12)

$$
\begin{gathered}
\left\|S_{M}^{0}-S_{N}^{0}\right\|_{2, U\left(R_{0}\right)}^{2} \leqq C \sum_{N+1}^{M}\left(\left\|\alpha_{n}^{*}\right\|^{2}(R)+\left\|\beta_{n}^{*}\right\|^{2}(R)+\left\|\gamma_{n}^{*}\right\|^{2}(R)\right) \\
+C \sum_{N+1}^{M}\left(\left\|a_{n}(r, \lambda)\right\|^{2}(R)+\left\|b_{n}(r, \lambda)\right\|^{2}(R)+\left\|c_{n}(r, \lambda)\right\|^{2}(R)+\left\|b_{n}^{\prime}(r, \lambda)\right\|_{0,\left(R_{0}, R_{1}\right)}^{2}\right) .
\end{gathered}
$$

Weil man für die ganze Summe $\sum_{n=1}^{\infty}\left(\left\|\alpha_{n}^{*}\right\|^{2}(R)+\ldots+\left\|b_{n}^{\prime}(r, \lambda)\right\|_{0,\left(R_{0}, R_{1}\right)}^{2}\right)$ die Schranke

$$
\leqq C\left(\|F-\lambda E\|^{2}+\|E\|^{2}+\|\nabla \times E\|^{2}\right) \leqq C(\lambda)\left(\|E\|^{2}+\|F\|^{2}\right)
$$

findet, ist $\left(S_{N}^{0}\right)_{1}^{\infty}$ als Cauchy-Folge konvergent. Für das Grenz-element $W_{\lambda} \in H^{2}\left(U\left(R_{0}\right)\right)^{3}$ hat man wegen (3.10) die Ungleichung (3.6). Die Behauptung (3.8) folgt aus (2.11).

Wir kehren nun zu der Aufgabe (1.1) mit $\lambda \in C, \lambda \neq 0$ und mit $\operatorname{div} F \in H_{0}^{1}(\Omega)$ zurück. Wie üblich zerlegen wir das Feld $F$ in einen divergenzfreien Teil $F_{0}$ und in ein Gradientenfeld $F_{1}$ wie folgt: $F=F_{0}+F_{1}, F_{1}=\nabla\left(\Delta_{0}^{-1}(\operatorname{div} F)\right)$. Wenn man die Lösung $E=E_{0}+E_{1}$ ähnlich darstellt, ist die Aufgabe (1.1) äquivalent mit

$$
\begin{gathered}
\nabla \times \nabla \times E_{0}+\lambda E_{0}=F_{0}, \quad E_{0} \in R^{0}(\Omega) \cap D_{0}(\Omega) \\
E_{1}=\lambda^{-1} F_{1} .
\end{gathered}
$$

Hier gelten die Abschätzungen

$$
\begin{aligned}
& \left\|E_{1}\right\| \leqq C(\lambda)\|\operatorname{div} F\|, \\
& \left\|E_{0}\right\| \leqq C(\lambda)(\|E\|+\|\operatorname{div} F\|) .
\end{aligned}
$$


Um den Teil $E_{1}$ behandeln zu können, brauchen wir eine Regularitätsaussage für die Poissonsche Aufgabe. Es liegt in unserem Interesse das Lemma in allen Dimensionen $p \geqq 2 \mathrm{zu}$ formulieren. Im folgenden sind die Funktionen $a_{n}$ die Entwicklungskoeffizienten in $u=\sum_{n=1}^{\infty} a_{n}(r) E_{n}(\omega)$. Ferner setzt man

$$
L_{n} u=-\left(u^{\prime \prime}+(p-1) r^{-1} u^{\prime}-\lambda_{n, 0} r^{-2} u\right) \text {. }
$$

3.2. Lemma. Sei (3.2i) erfüllt und sei $\lambda_{n, 0} \neq 4^{-1}\left(16-(p-2)^{2}\right)$ für alle $n$. Ferner sei $u, f \in H^{1}(U(R)), \Delta u=f$ sowie $\xi u, \xi f \in H_{0}^{1}(U(R))$ für $\xi \in \mathscr{D}(B(0, R))$. Für die Funktionen $u$ gilt in $U\left(R_{0}\right)$ die Zerlegung

$$
u=\sum_{\lambda_{n, 0}<\lambda(p)} \delta_{n} r^{v_{n}} E_{n}(\omega)+w
$$

mit $\lambda(p)=4^{-1}\left(16-(p-2)^{2}\right), v_{n}=2^{-1}\left(2-p+\left((2-p)^{2}+4 \lambda_{n, 0}\right)^{1 / 2}\right)$,

$$
\delta_{n}=\left((2-p)^{2}+4 \lambda_{n, 0}\right)^{-1 / 2} \int_{0}^{R} r^{-v_{n}+1} L_{n}\left(\eta a_{n}\right) d r .
$$

Ferner gilt $w \in H^{3}\left(U\left(R_{0}\right)\right)$, und man kann abschätzen

$$
\sum_{\lambda_{n, 0}<\lambda(p)} \delta_{n}^{2}+\|w\|_{3, U\left(R_{0}\right)}^{2} \leqq C\left(\|u\|_{1, U(R)}^{2}+\|f\|_{1, U(R)}^{2}\right) .
$$

Auf die Einzelheiten des Beweises, der wie Satz 3.1 über Lemma 2.1 läuft, wollen wir verzichten. Man beachte jedoch, daß die Voraussetzung $\xi f \in H_{0}^{1}(U(R))$ erforderlich ist, denn daraus folgert man, daß für $f=\sum_{n=1}^{\infty} \alpha_{n}(r) E_{n}$ der Gradient $\nabla f$ eine in $L^{2}\left(U\left(R_{1}\right)\right)^{p}, R_{1}<R$ konvergente Entwicklung

$$
\nabla f=\sum_{n=1}^{\infty} \alpha_{n}^{\prime} E_{n} \bar{e}_{r}+\sum_{n=1}^{\infty} r^{-1} \alpha_{n} \nabla_{\omega} E_{n}
$$

mit $\|\nabla f\|_{0, U\left(R_{1}\right)}^{2}=\sum_{n=1}^{\infty} \int_{0}^{R_{1}} r^{p-1}\left(\left|\alpha_{n}^{\prime}\right|^{2}+\lambda_{n, 0} r^{-2}\left|\alpha_{n}\right|^{2}\right) d r$ zuläßt. Ferner gilt $L_{n} a_{n}=$ $-\alpha_{n}$, und man spaltet die Koeffizienten $a_{n}$ nach Lemma 2.1 mit $\alpha=p+1, k=2$, $\alpha_{1}=p-1, k_{1}=1$ auf. Dieses Resultat kann man auch aus [4] herleiten.

Den folgenden Satz erhält man, wenn man Satz 3.1 auf den Teil $E_{0}$ anwendet, und den Teil $\nabla u, u=\lambda^{-1} \Delta_{0}^{-1}(\operatorname{div} F)$ nach dem obigen Lemma zerlegt. Die Konstanten $\tilde{\delta}_{0}^{i}$ und die Reihenentwicklung für $W_{\lambda}$ sind aus dieser Addition abzulesen.

3.3. Satz. Sei $\lambda \in C, \lambda \neq 0$ und sei $E \in R^{0}(\Omega) \cap D(\Omega)$ eine Lösung der Aufgabe (1.1) mit div $F \in H_{0}^{1}(\Omega)$. Ferner seien die Voraussetzungen (Vo 1)-(Vo 5) erfüllt, und es gelte $\lambda_{n, 0} \neq 15 / 4, \lambda_{n, 1} \neq 3 / 4$ für alle $n$. Dann besitzt das Feld $E$ in $L^{2}\left(U\left(R_{0}\right)\right)^{3}$ die Darstellung

$$
\begin{gathered}
E=\sum_{\lambda_{n, 0}<15 / 4} r^{-3 / 2+\left(1+4 \lambda_{n, 0}\right)^{1 / 2} / 2}\left(\tilde{\delta}_{n}^{1}(\lambda) E_{n}^{0}+\tilde{\delta}_{n}^{2}(\lambda) E_{n}^{1}\right) \\
\quad+\sum_{\lambda_{n}}{ }_{1}^{<3 / 4} r^{-1 / 2+\left(1+4 \lambda_{n, 1}\right)^{1 / 2} / 2}\left(\tilde{\delta}_{n}^{3} H_{n}^{2}\right)+\tilde{W}_{\lambda}
\end{gathered}
$$


mit $\tilde{W}_{\lambda} \in H^{2}\left(U\left(R_{0}\right)\right)^{3}$ derart, daß die Ungleichung

$$
\begin{gathered}
\left\|\tilde{W}_{\lambda}\right\|_{2, U\left(R_{0}\right)}^{2}+\sum_{\lambda_{n, 0}<15 / 4}\left(\left|\tilde{\delta}_{n}^{1}\right|^{2}+\left|\tilde{\delta}_{n}^{2}\right|^{2}\right)+\sum_{\lambda_{n, 1}<3 / 4}\left|\tilde{\delta}_{n}^{3}\right|^{2} \\
\leqq C(\lambda)\left(\|E\|_{0, \Omega}^{2}+\|F\|_{0, \Omega}^{2}+\|\operatorname{div} F\|_{1, \Omega}^{2}\right)
\end{gathered}
$$

gilt.

Das magnetische Feld $H$ besitzt im allgemeinen nicht so gute Regularitätseigenschaften wie $E$, weil $H$ keine Randbedingungen zu erfüllen braucht. Eine - auch physikalisch motivierte - Randbedingung für $H$ ist $\left.n \cdot H\right|_{\Gamma}=0$. Unter dieser Randbedingung kann man ähnliche Resultate für $H$ herleiten. Auf diese Frage wollen wir aber nicht näher eingehen.

\section{Literatur}

[1] Agmon, S.: Lectures on elliptic boundary value problems. - Van Nostrand Mathematica Studies 2. D. Van Nostrand Company, Inc., Princeton, New Jersey-TorontoNew York-London, 1965.

[2] Courant, R.: Ein allgemeiner Satz zur Theorie der Eigenfunktionen selbstadjungierter Differentialausdrücke. - Nachrichten von der Gesellschaft der Wissenschaften zu Göttingen, Mathematisch-physikalische Klasse aus dem Jahre 1923, Berlin, Weidmannsche Buchhandlung, 1924, 81-84.

[3] Courant, R., and D. Hilbert: Methods of mathematical physics. Vol. I. - Interscience Publishers, Inc., New York, 1965.

[4] Kondrat'ev, V. A.: Boundary problems for elliptic equations in domains with conical or angular points. - Trans. Moscow Math. Soc. 16, 227-313, 1967.

[5] LeIs, R.: Zur Theorie elektromagnetischer Schwingungen in anisotropen inhomogenen Medien. - Math. Z. 106, 1968, 213-224.

[6] LeIs, R.: Zur Theorie der zeitunabhängigen Maxwellschen Gleichungen. - Ber. Ges. Math. Datenverarb. Bonn 50, 1971,.

[7] LeIs, R.: Vorlesungen über partielle Differentialgleichungen zweiter Ordnung. - Bibliographisches Institut Mannheim, 1967.

[8] Mehra, M. L.: Zur asymptotischen Verteilung der Eigenwerte des Maxwellschen Randwertproblems. - Dissertation, Bonn, 1978.

[9] PICARD, R.: Zur Theorie der zeitunabhängigen Maxwellschen Gleichungen mit der Randbedingung $n(\nabla \times E)=n(\nabla \times H)=0$ im homogenen, anisotropen Medium. - Bonn. Math. Schr. 65, 1973, 1-76.

[10] Picard, R.: Zur Theorie der harmonischen Differentialformen. - Sonderforschungsbereich 72 ,Approximation und Optimierung“, preprint 150, 1977.

[11] Rinkens, H.-D.: Zur Theorie der Maxwellschen Gleichungen in der Ebene. - Bonn. Math. Schr. 38, 1969, 1-49.

[12] Saranen, J.: Über das Verhalten der Lösungen der Maxwellschen Randwertaufgabe in Gebieten mit Kegelspitzen. - Math. Methods Appl. Sci. 2, 1980, 235-250.

[13] SARANen, J.: Finite element method for the electric field of Maxwell's boundary value problem in polygonal domains of plane. - Erscheint demnächst.

[14] Steinbach, E.: Singuläre Lösungen zu Randwertproblemen der Maxwellschen Gleichungen. Diplomarbeit, Bonn, 1970. 
[15] Strang, G., and G. J. Fix: An analysis of the finite element method. - Prentice-Hall, Inc., Englewood Clifts, N. J., 1973.

[16] WeCK, N.: Eine Lösungstheorie für die Maxwellschen Gleichungen auf Riemannschen Mannigfaltigkeiten mit nichtglattem Rand. - Habilitationsschrift, Bonn, 1972.

[17] WeCK, N.: Maxwell's boundary value problem on Riemannian manifolds with nonsmooth boundaries. - J. Math. Anal. Appl. 46, 1974, 410-437.

[18] Wilcox, C. H.: The steady-state diffraction of electromagnetic radiation by an obstacle in an homogeneous anisotropic conducting medium. - Arch. Rational Mech. Anal. 14, 1963, 326-336.

Universität Jyväskylä

Mathematisches Institut

Sammonkatu 6

SF-40100 Jyväskylä 10

Finnland

Eingegangen am 2. Januar 1980 